\title{
Contribuições da Análise do Comportamento para a Educação Especial em periódicos brasileiros no período de 2008 a 2018
}

\author{
Contributions from Behavior Analysis to Special Education in Brazilian \\ journals from 2008 to 2018
}

Contribuciones del Análisis del Comportamiento para la Educación Especial en periódicos brasileños en el período de 2008 a 2018

\section{Rafael Ernesto Arruda Santos}

Doutorando pela Universidade Federal de São Carlos, São Carlos, São Paulo, Brasil.

rafael_ernesto_arruda_santos@hotmail.com

ORCID - https://orcid.org/0000-0002-8078-2633

\section{Nassim Chamel Elias}

Professor doutor pela Universidade Federal de São Carlos, São Carlos, São Paulo, Brasil. nchamel@terra.com.br

ORCID - https://orcid.org/0000-0003-4197-623X

Recebido em 14 de maio de 2019

Aprovado em 29 de julho de 2019

Publicado em 12 de novembro de 2019

\section{RESUMO}

A relação da Análise do Comportamento com a Educação Especial é evidenciada por livros, artigos e temas de pesquisa elaborados por pesquisadores internacionais e nacionais. Entretanto, a maioria das revisões de literatura e revisões sistemáticas foca seus objetivos no Transtorno do Espectro do Autismo (TEA). Nesse contexto, o objetivo desse trabalho foi verificar o impacto dos princípios da Análise do Comportamento em intervenções com indivíduos que pertencem ao público-alvo da Educação Especial utilizando uma revisão sistemática em artigos empíricos produzidos em revistas brasileiras da área no período de 2008 a 2018. O método consistiu de pesquisas no campo de busca das revistas nacionais de análise do comportamento com termos chaves, leitura do título e resumo para exclusão e inclusão dos artigos, armazenamento dos artigos incluídos e leitura completa do texto para análise qualitativa e quantitativa. Os resultados apresentam 39 artigos, sendo 18 artigos sobre surdez/deficiência auditiva, 18 sobre TEA e 3 sobre deficiência intelectual. Os métodos experimentais prevalecem na literatura, principalmente com delineamentos que tem o sujeito como seu próprio controle. Os estudos sobre surdez apresentam dois grupos distintos: implante coclear e uso de Libras. Os estudos sobre TEA apresentam um aumento ao longo dos anos e uma ênfase nos pais como educadores da criança. Dessa forma, os pesquisadores de Análise do Comportamento têm se especializado em públicos como deficientes auditivos e TEA, mas há uma lacuna na área com outros públicos, como surdocegos e altas habilidades/superdotação, sendo esta análise importante para pesquisas futuras da área.

Palavras-chave: Avaliação; Atendimento educacional especializado; Sala de recursos multifuncionais. 
http://dx.doi.org/10.5902/1984686X38150

\section{ABSTRACT}

The relationship between Behavior Analysis and Special Education is evidenced by books, published papers, and research program developed by international and national researchers. However, the majority of literature and systematic reviews focuses in Autistic Spectrum Disorder (ASD) research. In this context, the study objective was to verify Behavior Analysis principles impact on interventions with individuals who belong to the Special Education public using a systematic review of empirical articles produced in Brazilian journals between 2008 and 2018. The method consisted of searching national Behavior Analysis journals with key terms, reading the title and abstract for exclusion and inclusion of papers, storage of papers included and complete reading of the text for qualitative and quantitative analysis. Results present 39 papers, being 18 papers on deafness / hearing impairment, 18 on ASD and three on intellectual disability. Experimental methods prevail in literature and presenting data about the subject as its own control. Studies on deafness present two distinct groups: individuals with cochlear implant and individuals who use sign language. Studies on ASD have shown an increase over the years and an emphasis on parents as the child's educating intervener. Thus, Behavior Analysis researchers have specialized in audiences such as individuals with hearing impairment and with ASD, but there is a lack in the field with other audiences, such as deafblind and high skills / gifted individuals. This analysis is important for future research in the area.

Keywords: Behavior Analysis; Special Education; Knowledge Production.

\section{RESUMEN}

La relación entre el Análisis del Comportamiento y la Educación Especial se evidencia en la investigación internacional y nacional. Sin embargo, la mayoría de las revisiones bibliográficas se centran en el trastorno del espectro autista (TEA). En este contexto, el objetivo de este trabajo fue verificar el impacto de los principios de Análisis de la Conducta en las intervenciones con personas del público objetivo de Educación Especial mediante una revisión sistemática de artículos empíricos producidos en revistas brasileñas de 2008 a 2018. El método Consistió en búsquedas en revistas nacionales de análisis de comportamiento con términos clave, lectura de títulos y resumen para la exclusión e inclusión de artículos, almacenamiento de artículos incluidos y lectura completa del texto para el análisis cualitativo y cuantitativo. Los resultados presentan 39 artículos, 18 artículos sobre sordera / pérdida auditiva, 18 sobre TEA y tres artículos sobre discapacidad intelectual. Los métodos experimentales prevalecen en la literatura, especialmente con diseños que tienen el tema como su propio control. Los estudios sobre sordera presentan dos grupos distintos: implantación coclear y uso de Libras. Los estudios sobre el TEA han aumentado con los años y un énfasis en los padres como educadores de niños. Por lo tanto, los investigadores de Análisis del Comportamiento se han especializado en audiencias como personas con problemas de audición y TEA, pero existe una brecha con otras audiencias como sordociegos y habilidades altas / talentosas, y este análisis es importante para futuras investigaciones.

Palabras clave: Evaluación; Atención educativa especializada; Sala de recursos multifunción. 
http://dx.doi.org/10.5902/1984686X38150

\section{Introdução}

Segundo a Lei de Diretrizes e Bases da Educação Nacional (BRASIL, 1996, p. 19), que estabelece as diretrizes da educação nacional, a Educação Especial é definida como "a modalidade de educação escolar oferecida preferencialmente na rede de ensino regular", ou seja, uma modalidade dentro da educação regular focada no atendimento de um público-alvo específico, a saber "educandos com deficiência, transtornos globais do desenvolvimento e altas habilidades ou superdotação".

A diversidade no contexto do público-alvo da educação especial (PAEE) promove uma instituição de ensino com a necessidade de currículos, métodos, técnicas, recursos educativos e organizações específicas (BRASIL, 1996). Ainda nessa Lei, a diversidade desse PAEE implica na especialização de profissionais, reflexões de políticas educacionais, acesso igualitário e integração na comunidade por meio da profissionalização.

Neste contexto de diversidades do PAEE, os métodos e propostas teóricas visando melhorias educacionais e de qualidade de vida são amplos. Por exemplo, Nunes (2014) apresenta, em cada capítulo, um método diferente embasado por reflexões teóricas específicas, como Pesquisa Experimental, Metodologia de História de Vida, Etnografia e Pesquisa-ação.

No capítulo sobre Pesquisa Experimental (NUNES; WALTER, 2014), os autores apresentam vários delineamentos experimentais de sujeito único, uma especificidade comum nas pesquisas de Análise do Comportamento (COZBY; BATES, 2011). No mesmo caminho, alguns livros clássicos da Análise do Comportamento apresentam uma correlação com a Educação Especial, como é o caso do livro Single Subject Research Methodology in Behavioral Sciences (GAST, 2010). Neste livro, os delineamentos experimentais apresentados são importantes para resolução aplicada de situações educacionais e também na educação especial.

A relação entre a Análise do Comportamento e a Educação Especial é comprovada também por várias revisões sistemáticas envolvendo análise de artigos sobre Transtorno do Espectro do Autismo (GOULART; ASSIS, 2002; GUERRA; ALMEIDA-VERDU, 2016; MARTES; SANTOS-CARVALHO, 2012), implante coclear (CEDRO; PASSARELLI; HUZIWARA, 2014), sobre ensino de operantes verbais em periódico internacional (ESTEVES; LUCHESSI; ALMEIDA-VERDU, 2014), sobre a produção de Analistas do Comportamento em periódico internacional sobre Educação Especial (NICOLINO; 
http://dx.doi.org/10.5902/1984686X38150

ZANOTTO, 2010) e sobre Educação e Análise do Comportamento em periódicos nacionais (NICOLINO; ZANOTTO, 2011).

O estudo de Nicolino e Zanotto (2010) teve como objetivo analisar os trabalhos envolvendo o PAEE, no período de 2001 a 2008, do Journal of Applied Behavior Analysis (JABA). As variáveis analisadas no estudo foram: categoria do público-alvo, quantidade de participantes, tipo de pesquisa, delineamento, agente da mudança comportamental, observador, ambiente, medida, procedimento de intervenção, tema, objetivo e resultados. Os autores apresentam 46 artigos, sendo 27 sobre autismo (58\%), seguidos em menor escala por deficiência intelectual (ou retardo mental, por alguns artigos serem mais antigos) (8\%) e deficiência física (8\%). Outros dados importantes apresentados por Nicolino e Zanotto (2010) são: a predominância do modelo experimental, o pesquisador sendo o mais presente como agente da mudança/observação comportamental, o ambiente com maior ocorrência é a escola do participante e os temas mais predominantes são controle de estímulos específicos sobre repertórios e linguagem.

Na mesma direção, a revisão de Esteves, Luchessi, e Almeida-Verdu (2014) sobre o ensino de ecoico, mando e tato no JABA, entre 1968 a 2012, apresentou a mesma prevalência de estudos sobre autismo. Vale ressaltar que os autores apresentam uma porcentagem de $4 \%$ dos estudos com foco em participantes com deficiência auditiva.

No cenário nacional, Nicolino e Zanotto (2011) analisaram trabalhos sobre a Análise do Comportamento e a Educação, no período de 1961 a 2006. Os autores analisaram revistas de Psicologia e específicas da Análise do Comportamento - Cadernos de Análise do Comportamento, Revista Brasileira de Terapia Comportamental e Cognitiva (RBTCC) e Revista Brasileira de Análise do Comportamento (REBAC) - procurando termos técnicos, conceitos ou temas da área. Foram selecionados 71 trabalhos. Dentro dessa quantidade, 17 artigos eram específicos da Educação Especial (24\%). Os temas mais pesquisados dentro da Educação Especial eram sobre habilidades acadêmicas, linguagem e habilidade de ensino. Em geral, os participantes tinham até cinco anos de idade. Os autores não especificam qual o PAEE envolvido nos estudos analisados.

Os estudos focados em públicos específicos, indivíduos com autismo ou que usam implante coclear, apresentam dados parciais sobre a realidade geral da Educação Especial, como também seu baixo enfoque em produções nacionais. $O$ artigo de Nicolino e Zanotto (2011) apresenta dados nacionais da Educação como modalidade geral, 
http://dx.doi.org/10.5902/1984686X38150

trazendo as análises da Educação Especial como categoria comparativa à Educação Regular.

Nesse contexto, o objetivo desse trabalho foi verificar o impacto dos princípios da Análise do Comportamento em intervenções com indivíduos que pertencem ao públicoalvo da Educação Especial utilizando uma revisão sistemática em artigos empíricos produzidos em revistas brasileiras da área ${ }_{2}$ no período de 2008-2018. Mais especificamente, analisar: número de participantes e a qual categoria do PAEE eles pertencem, ambiente, delineamento experimental, procedimento de intervenção e análise qualitativa.

\section{Método}

Foram analisados os artigos publicados entre 2008 e 2018, das seguintes revistas brasileiras de Análise do Comportamento: Revista Brasileira de Terapia Comportamental e Cognitiva (RBTCC), Revista Brasileira de Análise do Comportamento (REBAC), Perspectivas em Análise do Comportamento e Revista Latina de Análise do Comportamento (ACTA COMPORTAMENTALIA).

O processo de pesquisa foi realizado nos próprios websites das revistas. Os termos utilizados para a pesquisa foram baseados em leis brasileiras como a Política Nacional de Educação Especial (BRASIL, 2014), Resolução oo 4 (BRASIL, 2009), Lei ㄲo 13.146 (BRASIL, 2015) e Lei oㅜ 12.764 (BRASIL, 2012); em pesquisa de termos relacionados no Thesaurus (Instituto Nacional de Estudos e Pesquisas Educacionais Anísio Teixeira INEP e Biblioteca Virtual em Saúde - BVS); e nos eixos temáticos do Congresso Brasileiro de Educação Especial (CBEE). Os termos foram divididos em categorias de termos relacionados para pesquisa e categorização dos resultados, sendo eles:

- Aluno com Altas Habilidades; Aluno Superdotado; Superdotação; Superdotado; Altas Habilidades.

- Deficiência Auditiva; Surdez; Distúrbios da Audição; Deficiente Sensorial; Implante Coclear; Surdo; Surdos.

- Deficiência Física; Deficiente Físico; Hemiplegia; Paraplegia.

- Deficiência Intelectual; Doença Mental; Pessoa com Deficiência Intelectual.

- Deficiência Visual; Baixa Visão.

- Deficiências Múltiplas; Pessoa com Deficiências Múltiplas.

- Surdocegueira; Surdocego; Pessoa com surdocegueira. 
http://dx.doi.org/10.5902/1984686X38150

Transtorno Global do Desenvolvimento; Autismo; Autismo Precoce; Síndrome de Asperger; Síndrome de Rett; Transtorno Desintegrativo da Infância; Transtornos Invasivos Sem Outra Especificação; Distúrbios Globais do Desenvolvimento; Crianças Autistas; Transtorno do Espectro do Autismo.

O operador booleano "OR" foi utilizado para expandir as buscas nos websites, porém, em algumas revistas, nenhum resultado foi encontrado. Para evitar esse viés, optou-se por utilizar cada termo separadamente. A primeira seleção dos artigos consistiu na remoção de artigos repetidos. A segunda seleção, pela leitura dos resumos, seguiu os seguintes critérios de elegibilidade: a) apresentação de algum dos termos do PAEE; b) publicado em português; c) não ser estudo de revisão de literatura ou de revisão sistemática, nem estudo teórico. Os resultados das buscas são apresentados na Figura 1.

Figura 1 - Fluxo da seleção dos estudos

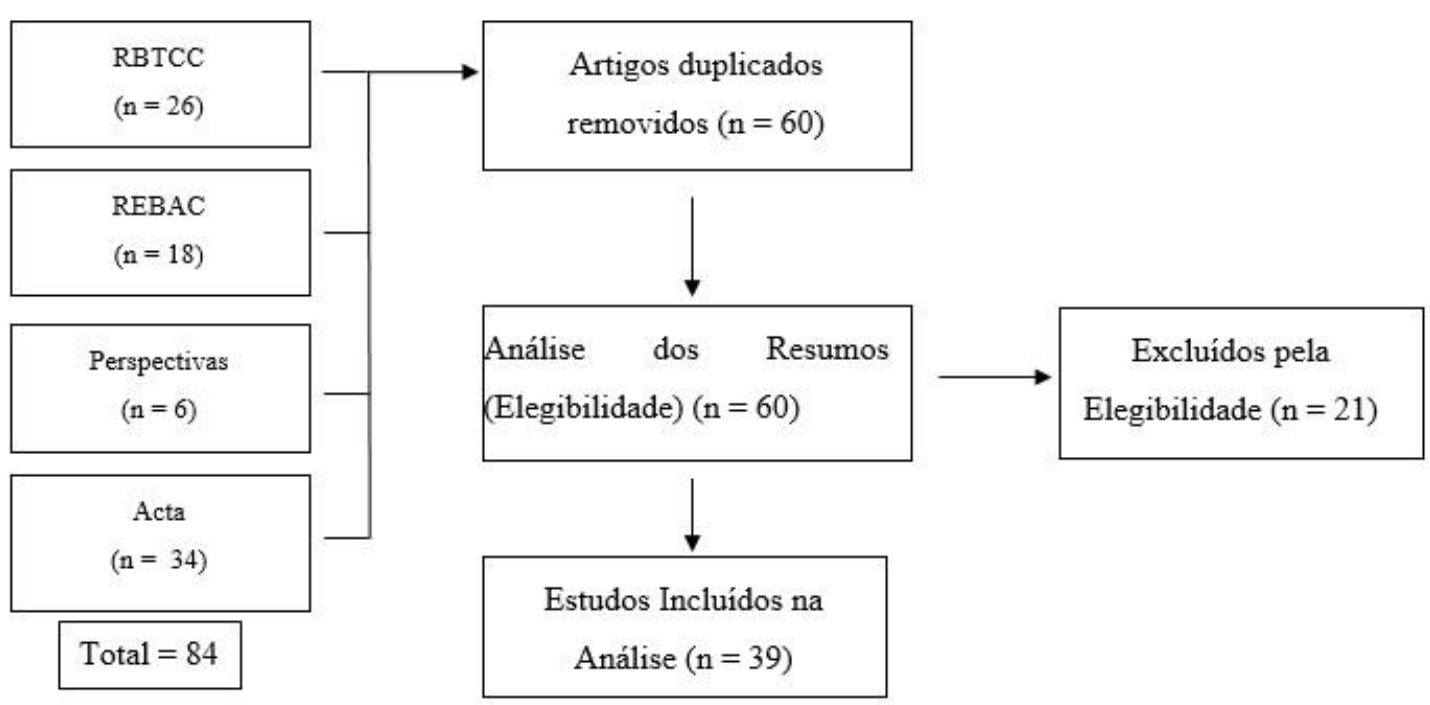

Fonte: Elaborado pelos autores.

As variáveis analisadas nos artigos foram baseadas, parcialmente, na pesquisa de Nicolino e Zanotto (2010) e nas diretrizes do Preferred Reporting Items for Systematic Reviews and Meta-Analyses (PRISMA; MOHER et al., 2009): a) Público-alvo, pautado nos termos chaves utilizados nas pesquisas e categorizados em oito grupos (Altas Habilidades; Surdez; Deficiência Física; Deficiência Intelectual; Deficiência Visual; Deficiência Múltipla; Surdocegueira; Transtorno do Espectro do Autismo); b) Cronologia das pesquisas por público-alvo; c) Número de participantes; d) Ambiente ou local; e) 
http://dx.doi.org/10.5902/1984686X38150

Delineamento da Pesquisa: experimental ou descritivo; f) Procedimento de Intervenção: aquisição de repertório, fortalecimento de repertório, controle de estímulos ou enfraquecimento de repertório; g) Análise Qualitativa (em que foram feitas as análises descritivas dos experimentos por público-alvo). As categorias para análise foram divididas em gerais (itens "a", "b" e "c") e específicas (itens "d", "e”, "f" e "g") para cada público-alvo encontrado nos artigos.

\section{Concordância entre observadores}

Essa concordância consistiu na pesquisa em espaços geográficos e temporais diferentes entre duas pessoas, seguindo as três etapas do fluxograma e conferindo os resultados das duas pesquisas. A etapa de pesquisa dos termos na revista e da remoção de artigos duplicados teve concordância de 100\%, porém o resultado a partir da leitura dos resumos teve uma concordância de $89,7 \%$ entre os observadores. A porcentagem mais baixa de concordância na leitura dos resumos ocorreu devido à revista RBTCC ter publicado uma edição especial sobre Transtorno do Espectro do Autismo (TEA). Dessa forma, a análise dos artigos empíricos dessa edição acarretou no aumento de quatro artigos para um dos observadores, fato ocorrido após o fechamento da concordância entre observadores.

\section{Resultados e Discussão}

Dos 39 artigos selecionados, 18 tinham como participantes indivíduos com surdez, 18 com TEA e 3 com Deficiência Intelectual. Não foi encontrado nenhum trabalho sobre a Análise do Comportamento nestas revistas para as outras categorias incluídas no PAEE. Dados semelhantes são apresentados por Nicolino e Zanotto (2010), em que, entre os estudos apresentados sobre educação especial no Journal of Applied Behavior Analysis (JABA), há uma prevalência de trabalhos com TEA, número três vezes maior do que o segundo colocado (Deficiência Intelectual). Uma diferença entre os dados encontrados nessa revisão e os encontrados por Nicolino e Zanotto (2010) está na prevalência de estudos com participantes com surdez em trabalhos nacionais, pois Nicolino e Zanotto (2010) não apresentam nenhum estudo nessa área. Essa diferença pode ser função da diferença entre os termos pesquisados em ambos os estudos. Também em relação à surdez, a revisão bibliográfica realizada por Esteves, Lucchesi e Almeida-Verdu (2014) no JABA indica uma baixa produção sobre artigos relacionados a tato, mando e ecoico para 
http://dx.doi.org/10.5902/1984686X38150

pessoas com surdez. Tal dado corrobora a baixa prevalência no campo internacional sobre análise do comportamento e deficiência auditiva.

A Figura 2 apresenta a quantidade de artigos publicada por ano no período investigado. É possível observar dois fenômenos nas publicações: um crescimento nas pesquisas sobre TEA e uma tradição de pesquisa sobre surdez. No caso do TEA, os estudos iniciam em 2011, sendo que em 2018 há o maior número de publicações. Esta taxa elevada em 2018 é devida, em parte, pela publicação de uma edição especial sobre Análise do Comportamento Aplicada (ABA) ao TEA na RBTCC. Em relação à surdez, o primeiro estudo aparece em 2008, mantendo pelo menos uma publicação até 2018 (com exceção de 2014), o que mostra um padrão de publicação com maior ocorrência em 2016. No estudo de Nicolino e Zanotto (2010), não se observa nenhuma tendência nas categorias específicas ao longo dos anos, porém os autores citam a junção entre TEA e tecnologias comportamentais como principal fator da prevalência do TEA nos estudos. Ao comparar com os dados nacionais, é possível inferir esse crescimento de estudos com TEA, como também uma tradição de estudos com surdez, sendo essa tecnologia crescente para ambos os públicos.

Figura 2 - Quantidade de artigos de acordo com o ano de publicação

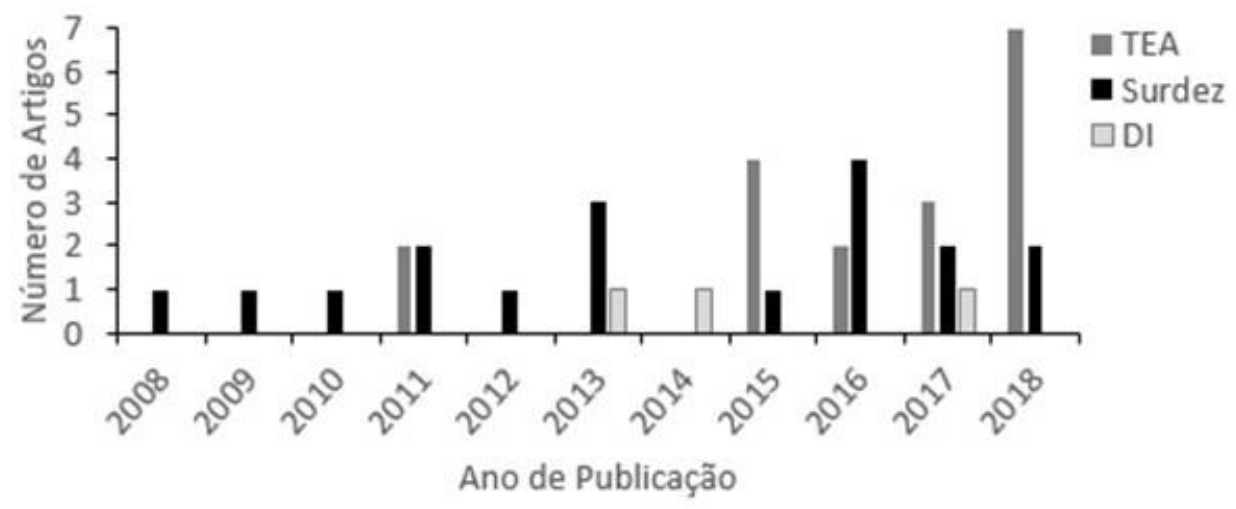

Fonte: Elaborado pelos autores.

A Figura 3 apresenta os dados sobre a quantidade de participantes nos estudos analisados. A maioria dos estudos apresenta de três a seis participantes. Entretanto, alguns experimentos apresentam de 12 até 23 participantes. Analisando estes artigos com 12 ou mais participantes é possível constatar o uso de delineamentos com grupos experimentais (os detalhes sobre esses experimentos estão nas análises qualitativas das categorias deficiência intelectual e surdez apresentadas a seguir). 
http://dx.doi.org/10.5902/1984686X38150

Figura 3 - Quantidade de artigos em relação à quantidade de participantes por categoria

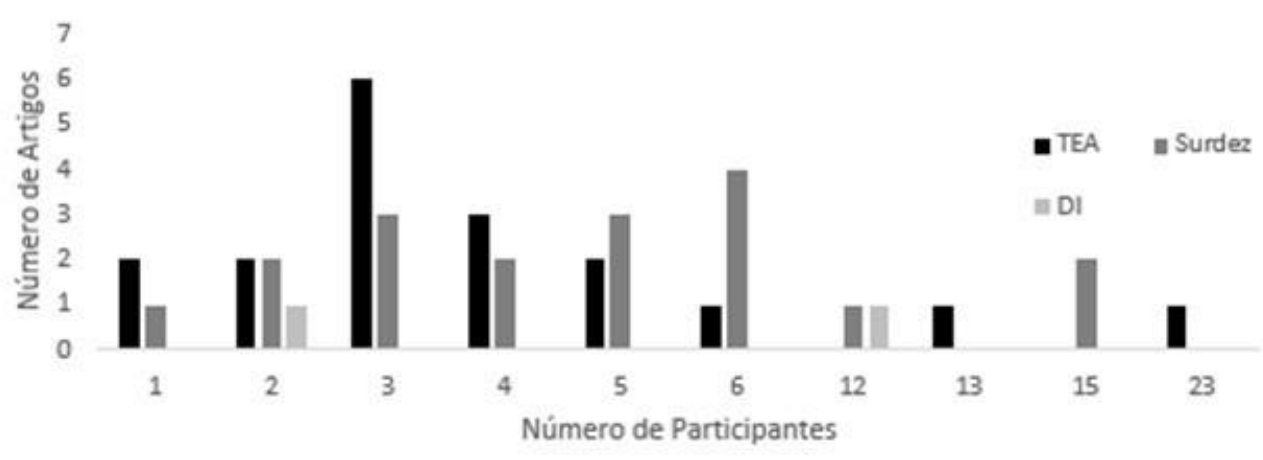

Fonte: Elaborado pelos autores.

Os dados relacionados ao número de participantes apresentam semelhanças com outras revisões sobre Educação Especial no JABA (NICOLINO; ZANOTTO, 2010), comportamento verbal e TEA no JABA (MARTES; SANTOS-CARVALHO, 2012) e operantes verbais e TEA no The Analysis of Verbal Behavior (GUERRA; ALMEIDAVERDU, 2016). Nicolino e Zanotto (2010) e Martes e Santos-Carvalho (2012) enfatizam a abordagem com o delineamento de sujeito único como principal fator para o número de participantes entre um e quatro em detrimento aos delineamentos estatísticos ou de grupos. Na revisão de Guerra e Almeida-Verdu (2016), é possível identificar uma variação maior na quantidade de participantes, de um a oito participantes, sendo a maioria com um a três participantes. Dessa forma, os dados indicam prevalência do delineamento de sujeito único nos estudos nacionais e internacionais que versam sobre Análise do Comportamento e o PAEE.

A análise do número de participantes revelou três grupos de estudos envolvendo participantes: grupos homogêneos, em que há somente participantes da mesma categoria; grupos heterogêneos, em que há participantes do PAEE e participantes com desenvolvimento típico; grupos agregados, em que cuidadores ou profissionais eram os participantes. Foram encontrados 27 artigos com grupo homogêneo, sete com grupo agregado e cinco com grupo heterogêneo. Os estudos envolvendo crianças ou adolescentes com desenvolvimento típico avaliam 0 desempenho destes com participantes do PAEE; por exemplo, estudos comparando o desempenho de surdos com ouvintes (SANCHEZ; GOUVEIA, 2008; SANCHEZ; GOUVEIA, 2011), TEA e desenvolvimento típico (NICOLINO; MALERBI, 2011), TEA, deficiência intelectual e 
http://dx.doi.org/10.5902/1984686X38150

desenvolvimento típico (GARCIA; ZAVITOSKI; ELIAS, 2018), e desenvolvimento intelectual e desenvolvimento típico (MALERBO; SCHMIDT, 2017). O grupo com participantes agregados envolvia a participação de cuidadores (BARBOZA et al., 2015; BORBA et al., 2015; FERREIRA; SILVA; BARROS, 2016; GUIMARÃES et al., 2018; MESQUITA et al., 2014; SILVA et al., 2017) e profissionais no atendimento de puericultura no Sistema Único de Saúde (SUS) (MURARI; MICHELLETO, 2018).

\section{Estudos com Participantes Surdos ou com Deficiência Auditiva}

Cinco estudos sobre surdez foram realizados em salas de escolas comuns (ELIAS; ANGELOTTI, 2016; MAGALHÃES; ASSIS; ROSSIT, 2016; PASSARELLI et al., 2013; RESENDE; ELIAS; GOYOS, 2012; SANTOS; ASSIS; BORBA, 2016), quatro em escolas especializadas (ASSIS et al. 2011; SANCHEZ; GOUVEIA, 2008; SANCHEZ; GOUVEIA, 2011; SOUZA et al., 2010), dois em salas de hospitais (BATTAGLINI; ALMEIDA-VERDU; BEVILACQUA, 2013; SILVA; NEVES; ALMEIDA-VERDU, 2017) e três em clínicas (HUSSEIN et al., 2018; LUCCHESI et al., 2015; SOUZA; ALMEIDA-VERDU; BEVILACQUA, 2013). Quatro estudos não apresentaram uma descrição clara do local de coleta (ALMEIDA-VERDU et al., 2009; ALMEIDA-VERDU; GOMES. 2017; MELO et alı, 2018; RIQUE et al:, 2017). Dessa forma, nota-se que os estudos apresentam aplicações em diversos contextos, considerando ainda as diferenças regionais da sua aplicação (Pará e São Paulo, por exemplo).

Entende-se, portanto, que a aplicação dos princípios comportamentais em vários contextos e com participantes com deficiência auditiva que usam implante coclear ou surdos usuários da Língua Brasileira de Sinais (LIBRAS) indica uma forte validade externa dos experimentos (VELASCO; GARCIA-MIJARES; TOMANARI, 2010). Ainda, seguindo a tradição analítico-comportamental, a utilização de (i) avaliações do repertório de entrada do participante (linha de base), (ii) intervenção e (iii) avaliação após a intervenção (pósteste) para verificar o efeito do ensino foi a característica comum na maioria dos estudos, independentemente das diferenças de locais, procedimento e forma de análise dos dados.

O delineamento de sujeito único é uma das características principais dos estudos selecionados. A definição de Gast (2010) inclui os delineamentos de sujeito único como experimentais, quantitativos, tendo o sujeito como seu próprio controle e a lógica da linha de base. Dessa forma, 88,8\% $(n=16)$ dos estudos com surdez verificaram a manipulação de variáveis independentes e quantificaram esses resultados, sendo que os participantes 
manipulavam e respondiam diante das contingências programadas e foram avaliados pelo seu repertório de entrada e resultado do experimento. Em contraste com essa porcentagem, estão os estudos de Sanchez e Gouveia $(2008,2011)$, nos quais o delineamento utilizado foi de grupo controle e experimental. Essa lógica experimental permeia também o estudo de Assis et al. (2011), pois apesar da inclusão de dois grupos no modelo experimental, os participantes foram analisados individualmente pela comparação dos desempenhos em pré e pós-testes. A diferença entre os dois grupos procurava avaliar o efeito da numerosidade explícita e implícita de estímulos não convencionais.

Em relação à intervenção utilizada, os estudos apresentam uma prevalência na utilização do procedimento de escolha de acordo com o modelo (MTS, do inglês matching-to-sample) e suas variações. As variações se deram nas configurações do procedimento de MTS com sobreposição de estímulos (ASSIS et al., 2011; SOUZA et al., 2010), ensino por exclusão (ALMEIDA-VERDU; GOMES, 2017; BATTAGLINI; ALMEIDAVERDU; BEVILACQUA, 2013; MELO et al., 2018), ensino de cópia (RESENDE; ELIAS; GOYOS, 2012), esvanecimento (SOUZA; ALMEIDA-VERDU; BEVILACQUA, 2013), escolha de acordo com o modelo por resposta construída (CRMTS, do inglês Constructed Response Matching-To-Sample) (MAGALHÃES; ASSIS; ROSSIT, 2016; SANTOS; ASSIS; BORBA, 2016) e tentativas discretas (RIQUE et al., 2017; SILVA; NEVES; ALMEIDA-VERDU, 2017).

Dessa forma, é possível concluir a forte evidência de validade externa na variação de contextos e locais dos experimentos de surdez, a predominância de experimentos com delineamento de sujeito único e também dos aspectos metodológicos e teóricos das intervenções sobre controle de estímulos.

As leituras dos artigos produziram a emergência de categorias qualitativas sobre posições, dados e discussões dos trabalhos em Análise do Comportamento para deficiência auditiva e surdez. O primeiro dado qualitativo é a influência dos trabalhos de Sidman (1994), Sidman e Tailby (1982), do paradigma de equivalência de estímulos e do uso do procedimento de MTS e suas variações. Todos os trabalhos apresentam uma referência ao autor e usam métodos para avaliar algumas de suas proposições, com exceção de Sanchez e Gouveia $(2008,2011)$.

Apesar dos pontos em comum, os estudos sobre deficiência auditiva e surdez apresentam uma separação em dois grandes grupos: (i) o grupo de estudos focados no 
http://dx.doi.org/10.5902/1984686X38150

público de crianças após a cirurgia para o Implante Coclear (ALMEIDA-VERDU et al., 2009; ALMEIDA-VERDU; GOMES, 2017; BATTAGLINI; ALMDEIA-VERDU; BEVILACQUA, 2013; HUSSEIN et al., 2018; LUCCHESI et al., 2015; MELO et al., 2018; PASSARELLI et al., 2013; RIQUE et al., 2017; SOUZA; ALMEIDA-VERDU; BEVILACQUA, 2013; SILVA; NEVES; ALMEIDA-VERDU, 2017) e (ii) o grupo de estudos concentrado no uso dos sinais em LIBRAS para o ensino de crianças surdas (ASSIS et al., 2011; ELIAS; ANGELOTTI, 2016; MAGALHÃES; ASSIS; ROSSIT, 2016; RESENDE; ELIAS; GOYOS, 2012; SANTOS; ASSIS; BORBA, 2016; SOUZA et al., 2010).

Lucchesi et al. (2015) apresentam uma definição mais refinada da lógica de ensino por trás do uso do implante coclear:

A abordagem aurioral privilegia a reabilitação auditiva por meio de dispositivos como o Aparelho de Amplificação Sonora Individual (AASI) e o Implante Coclear, e o ensino da linguagem oral, em detrimento ao ensino da linguagem de sinais e de leitura labial. (LUCCHESI et al., 2015, p.149)

Nesse contexto, os trabalhos realizam um treino do ouvir dos participantes uma vez que eles passaram pela cirurgia para o implante coclear. Alguns desses estudos apresentam resultados apontando para a interdependência entre o ouvir e o falar (ALMEIDA-VERDU et al., 2009; BATTAGLANI; ALMEIDA-VERDU; BEVILACQUA, 2013; SOUZA; ALMEIDA-VERDU; BEVILACQUA, 2013), enquanto outros estudos apresentam resultados sobre a aquisição da nomeação pelos participantes (ALMEIDA-VERDU; GOMES, 2017; HUSSEIN et al., 2018; RIQUE et al., 2017).

O grupo de estudos sobre surdez não apresenta uma definição de qual proposta de ensino dentro do contexto histórico da surdez ${ }^{1}$ foi adotada, mas todos utilizam a Língua Brasileira de Sinais (LIBRAS) como estímulo ou como forma de instrução nas suas configurações metodológicas. O estudo de Souza et al. (2010) foi o único com uma breve descrição da possibilidade de contexto de ensino e utilizou a LIBRAS e a comunicação total para se comunicar com as crianças surdas, porém não defende nenhuma proposta em particular. Os estudos desse grupo de pesquisadores apresentam dados sobre 0 ensino de matemática e português para crianças surdas.

Os estudos sobre questões da matemática baseados na análise do comportamento em crianças surdas apresentam dados sobre relações ordinais (SOUZA, et al., 2010; ASSIS et al., 2011), ensino de frações (ELIAS; ANGELOTTI, 2016) e relações monetárias (MAGALHÃES; ASSIS; ROSSIT, 2016). Dois estudos apresentam dados sobre o ensino de construção de sentenças em Português (RESENDE; ELIAS; GOYOS, 2012; SANTOS; 
http://dx.doi.org/10.5902/1984686X38150

ASSIS; BORBA, 2016). Os estudos de Sanchez e Gouveia (2008; 2011) apresentam uma particularidade que se refere à utilização de escalas para avaliação do público surdo usuário de LIBRAS, comparando com um grupo controle de ouvintes. Sanchez e Gouveia. (2008) trazem dados sobre a efetividade da Escala Analógica de Humor (EAH), adaptada em LIBRAS, enquanto Sanchez e Gouveia (2011) avaliaram a ansiedade em etapas da apresentação em público com surdos e ouvintes.

\section{Estudos com Participantes com TEA}

A categoria de estudos sobre o TEA também apresentou uma quantidade significativa para análise quantitativa e qualitativa. A primeira variável analisada foi o local de coleta dos dados. Oito estudos (BACELAR; NEVES; SOUZA, 2017; CALADO et al., 2018; BARBOZA et al., 2015; BORBA et al., 2015; DIAS; BARROS, 2017; FERREIRA; SILVA; BARROS, 2016; GUIMARÃES et al., 2018; SILVA et al., 2017) foram realizados em salas de atendimento a essa população em uma mesma universidade federal, dois estudos (NICOLINO; MALERBI, 2011; VARELLA; SOUZA, 2011) foram conduzidos na casa dos participantes, dois (FIALHO; MICHELETTO; SÉLIOS, 2015; GARCIA; OLIVEIRA, 2016) na sala de uma clínica, dois estudos (GARCIA; ZAVITOSKI; ELIAS, 2018; PLÁCIDO; MEDEIROS; CRUZ, 2018) em instituição especializada, três não definiram o local de coleta (CALADO et al.,2018; GOMES; HANNA; SOUZA, 2015; ROCANTI; HÜBNER, 2018) e um estudo coletou em uma Unidade Básica de Saúde (UBS) do SUS (MURARI; MICHELETTO, 2018). Esses dados sugerem uma tendência de pesquisa apresentada por uma pós-graduação ou serviço em especial. O surgimento dessa tendência e a informação do local podem colaborar para análises do mapeamento dos serviços e estudos focados nos aspectos geográficos e bibliográficos sobre temas específicos ou para a população procurar o serviço adequado. A variação dos estudos também colabora para a validade externa dos experimentos, conforme discutido nos resultados da categoria sobre surdez.

Em relação ao delineamento experimental, a maior parte dos estudos $(n=16)$ foi experimental, seguindo a tradição do delineamento de sujeito único com a utilização de pré-teste para avaliar os repertórios em linha de base, intervenção e pós-teste. As variações em relação ao modelo de pré-teste e pós-teste estão nos estudos que utilizaram grupo experimental e grupo controle (GARCIA; ZAVITOSKI; ELIAS, 2018) e estudo qualitativo observacional (MURARI; MICHELETTO, 2018). 
http://dx.doi.org/10.5902/1984686X38150

Os artigos com TEA têm grande variabilidade de intervenções comportamentais e de público-alvo, podendo ser divididos em três categorias principais: (i) equivalência de estímulos, (ii) intervenção com os cuidadores e (iii) temas variados. Seis estudos mantêm a tradição na utilização do procedimento de MTS (CALADO et al., 2018; CALLOU; ASSIS; BORBA, 2018; DIAS; BARROS, 2017; GOMES; HANNA; SOUZA, 2015; PLÁCIDO; MEDEIROS; CRUZ, 2018; VARELLA; SOUZA, 2011), e alguns utilizam também variações desse procedimento, como procedimento de MTS blocado (VARELLA; SOUZA, 2011), MTS com múltiplos exemplares (BARCELAR; NEVES; SOUZA, 2017; SILVA et al_, 2017) e a relação entre MTS e o procedimento de reversões repetidas de discriminação simples (DIAS; BARROS, 2017).

Alguns estudos utilizaram a modelação ao vivo para verificar o efeito na interação entre a criança com TEA e seus colegas (NICOLINO; MALERBI, 2011) e vídeo modelação para ensino de cuidadores de crianças com TEA (BARBOZA et al., 2015). Cinco estudos (BARBOZA et al., 2015; BORBA et al., 2015; FERREIRA; SILVA; BARROS, 2016; GUIMARÃES et al., 2018; SILVA et al., 2017) desenvolveram programas de intervenção focado nos cuidadores da criança com TEA, por meio de vídeo modelação, modelação, ensino com múltiplos exemplares, instrução, feedbacks e ensaios.

Outros estudos focaram em temas mais específicos que não criaram categorias abrangentes quanto à intervenção. Fialho, Micheletto e Sélios (2015) utilizaram jogos para avaliar questões sobre variabilidade comportamental. Garcia e Oliveira (2016) utilizaram reforçamento não-contingente e treino de mando para redução de comportamentos auto lesivos. Barcelar, Neves e Souza (2017) utilizaram procedimentos mesclados para avaliar pessoas como estímulo discriminativo e/ou reforçadores condicionados e seu efeito nos repertórios de atenção conjunta, tato e mando em crianças com TEA. Rocanti e Hübner (2018) utilizaram a manipulação da magnitude do reforçador no ensino de tato e discriminação auditivo-visual. Garcia, Zavitoski e Elias (2018) avaliaram a testagem de um protocolo de respostas intraverbais com três grupos distintos (TEA, DI e desenvolvimento típico). Murari e Micheletto (2018) não realizaram intervenção, mas observaram e registraram os dados na sala de uma UBS-SUS.

Cinco estudos sobre o TEA (CALADO et al., 2018; CALLOU; ASSIS; BORBA, 2018; DIAS; BARROS, 2017; GOMES; HANNA; SOUZA, 2015; VARELLA; SOUZA, 2011) estão 
http://dx.doi.org/10.5902/1984686X38150

em consonância com a análise qualitativa dos estudos com surdez, com uma quantidade significativa de estudos focados no paradigma da equivalência de estímulos.

Varella e Souza (2011) utilizaram o procedimento blocado e o aumento gradual dos estímulos modelos e de comparação, uma variação experimental do procedimento de MTS. Na mesma lógica de variação de procedimento, Gomes, Hanna e Souza (2015) utilizaram o MTS adaptado com vários estímulos modelos. Dias e Barros (2017) compararam diferentes procedimentos de ensino na expansão de classes de estímulos pela reversão repetida de discriminações simples e pelo MTS. Ainda no contexto da equivalência de estímulos, Callou, Assis e Borba (2018) utilizaram o MTS e procedimentos de correções para avaliar, analisar e modificar o controle restrito, enquanto Calado et al. (2018) utilizaram o CRMTS para avaliar a emergência de construção de sentenças em crianças com TEA.

Cinco estudos focaram na intervenção aos cuidadores de crianças com TEA (BARBOZA et al., 2015; BORBA et al., 2015; FERREIRA; SILVA; BARROS, 2016; GUIMARÃES et al., 2018; SILVA et al., 2017). Os procedimentos utilizados variaram de leitura dos programas (BORBA et al., 2015), análise de vídeos com aplicação (BORBA et al., 2015), demonstração dos programas entre monitores (BORBA et al., 2015; FERREIRA; SILVA; BARROS, 2016), role-play do cuidador com o monitor (BORBA et al., 2015; FERREIRA; SILVA; BARROS, 2016), demonstração do monitor com a criança (BORBA et al., 2015), tentativa supervisionada do cuidador com a criança (BORBA et al., 2015), vídeo-feedback (FERREIRA; SILVA; BARROS, 2016; GUIMARÃES et al., 2018) e ensino por tentativas discretas para os cuidadores (BARBOZA et al., 2017; FERREIRA; SILVA; BARROS, 2016; SILVA et al., 2017). Esses estudos com programas para ensino de cuidadores têm colaborado para uma diminuição no tempo de intervenção do pesquisador ou profissional, como também colaboram para um alcance maior de crianças, uma vez que a intervenção não foca na criança, mas no grupo de cuidadores. Ferreira, Silva e Barros (2016) concluem que a efetividade desse modelo de intervenção pode ser a implementação de políticas públicas no SUS.

Os estudos focados em temas variados abordam temas como estudo com pares de crianças (NICOLINO; MALERBI, 2011), utilização de jogos para verificar a variabilidade comportamental (FIALHO; MICHELETTO; SÉLIOS, 2015), comportamentos auto lesivos com reforço não-contingente e mando (GARCIA; OLIVEIRA, 2016), atenção conjunta (BARCELAR; NEVES; SOUZA, 2017), tradução e aplicação de questionários (GARCIA; 
http://dx.doi.org/10.5902/1984686X38150

ZAVITOSKI; ELIAS, 2018), efeito da magnitude do reforço no desempenho dos participantes (ROCANTI; HÜBNER, 2018), análise de regras específicas e gerais (PLÁCIDO; MEDEIROS; DA CRUZ, 2018) e observação do atendimento de puericultura por profissionais do SUS (MURARI; MICHELETTO, 2018).

\section{Estudos com Participantes com Deficiência Intelectual}

O local de realização da coleta de dois estudos foi a sala de uma clínica especializada em atendimento ao PAEE (MACEDO; ESCOBAL; GOYOS, 2013; MALERBO; SCHIMIDT, 2017), enquanto o terceiro estudo não apresentou definição do local (MESQUITA et al., 2014). Em relação ao delineamento, dois estudos são experimentais e utilizaram o delineamento de sujeito único (MACEDO; ESCOBAL; GOYOS, 2013; MALERBO; SCHMIDT, 2017) e um estudo descritivo (MESQUITA et al., 2014). O estudo de Malerbo e Schmidt (2017) contou com o maior número de participantes $(n=24)$, divididos em dois grupos: desenvolvimento típico $(n=12)$ e deficiência intelectual $(n=12)$. Apesar do número maior de participantes, este estudo utilizou o delineamento de sujeito único.

As intervenções apresentadas nos estudos experimentais não seguem um padrão, sendo que Macedo, Escobal e Goyos (2013) utilizaram material de fibra de média densidade (MDF) para apresentação de estímulos com variações em seu valor calórico e na magnitude; e Malerbo e Schmidt (2017) utilizaram o MTS e a aprendizagem por exclusão para ensinar relação entre nome-textura para crianças típicas e com deficiência intelectual. O estudo descritivo de Mesquita et al. (2014) apresentou dados da diferença entre dois grupos, um grupo sem restrição alimentar e outro com restrição alimentar, porém não houve intervenção e os dados foram coletados com instrumentos para análise qualitativa e quantitativa.

As categorias qualitativas emergentes nos estudos sobre deficiência intelectual são poucas. A primeira e mais destacável refere-se ao comportamento de alimentar-se, sendo avaliadas a escolha e a preferência baseadas nos valores calóricos (MACEDO; ESCOBAL; GOYOS, 2013) e o efeito da restrição alimentar em grupos de crianças com Síndrome de Prader-Willi (MESQUITA et al., 2014). Os resultados do estudo de Macedo, Escobal e Goyos (2013) indicam uma preferência por magnitude (quantidade de reforçadores) por um participante, mas nenhuma diferença em relação ao valor calórico dos alimentos. Os experimentadores discutem possíveis variáveis que afetam esses 
http://dx.doi.org/10.5902/1984686X38150

resultados: a igualdade do valor do reforço, considerado manipulável em próximos experimentos; a ausência de discriminabilidade nas tentativas, que também pode ser testada em outros estudos; ausência do repertório de escolha, o que poderia ser treinado antes da replicação do estudo; e variáveis filogenéticas (estas variáveis somente podem ser inferidas).

Da mesma forma, o estudo de Mesquita et al. (2014) avaliou o efeito da presença ou não de restrição alimentar e o ganho de peso em crianças com Síndrome de Prader-Willi. Os resultados mostraram o aumento do peso para o grupo sem restrição alimentar, e as crianças que passaram por restrição alimentar apresentaram mais comportamentos problemáticos. A conclusão principal dos autores está na eficácia dos procedimentos comportamentais em relação ao mal-uso da restrição alimentar, ou seja, intervenções comportamentais bem planejadas podem melhorar o controle do peso e diminuir os comportamentos problemas.

\section{Considerações finais}

O objetivo desse estudo foi analisar os artigos produzidos em revistas brasileiras de Análise do Comportamento sobre o público-alvo da Educação Especial, no período de 2008-2018. Os resultados retornaram 39 artigos empíricos com dados sobre a aplicação de princípios da Análise do Comportamento em participantes PAEE.

A prevalência de estudos sobre o TEA é um dado que corrobora outros estudos (ESTEVES; LUCHESSI; ALMEIDA-VERDU, 2014; MARTES; SANTOS-CARVALHO, 2012; NICOLINO; ZANOTTO, 2010). Em contrapartida, os dados apresentaram, na mesma quantidade, uma prevalência de estudos sobre deficiência auditiva/surdez, o que difere dos dados apresentados por Esteves, Luchessi e Almeida-Verdu (2014) e Nicolino e Zanotto (2010).

Outro dado importante é o aumento de produções sobre o TEA nos últimos quatro anos, como também a continuidade dos estudos sobre surdez. O número de participantes, com raras exceções, atesta a importância de experimentos com delineamento de sujeito único.

Dentro das categorias de análise qualitativa, os estudos sobre surdez apresentam dois compromissos educacionais diferentes, um com tradição aurioral e outro com uso da Libras (CAPOVILLA, 2000). Já os estudos sobre autismo apresentam uma variabilidade de temas, como também de enfoque no participante, aplicação dos procedimentos com as 
http://dx.doi.org/10.5902/1984686X38150

crianças e com os pais, tendência debatida por Goulart e Assis (2002) e Martes e SantosCarvalho (2012).

As limitações desse estudo podem ser apontadas por dois caminhos: o caminho do Analista do Comportamento e o caminho do Educador Especial. No contexto do Analista do Comportamento, a limitação desse estudo foi o enfoque nas revistas nacionais com produções em Análise do Comportamento. Consequentemente, outras revistas da Psicologia não foram incluídas e futuros estudos poderiam replicar esse estudo com a inclusão dessas revistas. No contexto do Educador Especial, a outra limitação desse estudo residiria no problema de nomenclatura do PAEE. O estudo de Nicolino e Zanotto (2011), por exemplo, apresenta termos que não foram encontrados nas Leis, no Thesaurus e nem nos Eixos do CBEE. Dessa forma, a utilização de palavras-chaves e o estabelecimento de "unitermos" para melhoria das pesquisas e análises tornam-se necessários (GUERRA; ALMEIDA-VERDU, 2016).

Dessa maneira, novos estudos podem colaborar para validade externa da Análise do Comportamento aplicada à Educação Especial, realizando a manutenção de temas já pesquisados e explorando novos temas, como Análise do Comportamento e Surdocegueira, ou Superdotação/Altas Habilidades. Assim como estabelecer novos programas de ensino e currículos para o PAEE.

\section{Referências}

ASSIS, Grauben. et al. Efeitos da Ordem de Ensino e da Transferência de Funções sobre Relações Ordinais em Surdos. Acta Comportamentalia, Guadalajara, pp. 43-63, mar. 2011. Disponível em: http://www.revistas.unam.mx/index.php/acom/article/view/28013 Acesso em 27 dez. 2018.

ALMEIDA-VERDU, Ana Claudia Moreira. et al. Imitação Vocal e Nomeação de Figuras em Deficientes Auditivos Usuários de Implante Coclear: Um Estudo Exploratório. Revista

Brasileira de Análise do Comportamento, Belém, 5, pp. 83-78, jan. 2009. Disponível em: https://periodicos.ufpa.br/index.php/rebac/article/view/722 Acesso em 27 dez. 2018.

ALMEIDA-VERDU, Ana Cláudia Moreira; GOMES, Fernanda Piqueira. Precisão da Fala em Nomeação de Figuras após Formação de Classes de Equivalência em Crianças com Implante Coclear. Revista Perspectivas em Análise do Comportamento, São Paulo, 2, pp;274-287, ago. 2017. Disponível em:

https://www.revistaperspectivas.org/perspectivas/article/view/203/189 Acesso em: 27 dez. 2018.

BACELAR, Flávia Teresa Neves Silva; NEVES, Elizabeth Bezerra; SOUZA, Carlos Barbosa Alves. Relações Funcionais entre Estímulos Condicionais Sociais, Atenção Conjunta, Tato e 
http://dx.doi.org/10.5902/1984686X38150

Mando em Crianças com Autismo. Revista Brasileira de Terapia Comportamental e Cognitiva, São Paulo, pp. 45-61, fev. 2017. Disponível em:

http://www.usp.br/rbtcc/index.php/RBTCC/article/view/1093/543 Acesso em: 28 dez. 2018.

BARBOZA, Adriano Alves. et al. Efeitos de Videomodelação Instrucional sobre o

Desempenho de Cuidadores na Aplicação de Programas de Ensino a Crianças

Diagnosticada com Autismo. Acta Comportamentalia, Guadalajara, pp. 45-421, dez. 2015.

Disponível em: http://www.revistas.unam.mx/index.php/acom/article/view/53794/47889

Acesso em: 28 dez. 2018.

BATTAGLINI, Maria Pavão; ALMEIDA-VERDU, Ana Claudia Moreira; BEVILACQUA, Maria Cecília. Aprendizagem via Exclusão e Formação de Classes Equivalentes em Crianças com Deficiência Auditiva e Implante Coclear. Acta Comportamentalia, Guadalajara, pp. 20-35, mar. 2013. Disponível em:

http://www.revistas.unam.mx/index.php/acom/article/view/36627/33188 Acesso em: 28 dez. 2018.

BORBA, Marilu Michelly Cruz de. et al. Efeito de Intervenção Via Cuidadores sobre Aquisição de Tato com Autoclítico em Crianças com TEA. Revista Brasileira de Análise do

Comportamento, Belém, pp. 15-23, jan. 2015. Disponível em:

https://periodicos.ufpa.br/index.php/rebac/article/view/3768/3800 Acesso em: 28 dez. 2018

BRASIL. Casa Civil. Lei no 9.394, de 20 de Dezembro de 1996. Brasília, DF, 1996. 28p.

BRASIL. Ministério da Educação. Resolução n4 de 2 de Outubro de 2009. Brasília, DF, 2009. 3p.

BRASIL. Casa Civil. Lei no 12.764, de 27 de Dezembro de 2012. Brasília, DF, 2012. 3p.

BRASIL. Ministério da Educação. Política Nacional da Educação Especial na Perspectiva da Educação Inclusiva. Brasília, DF, 2014. 15p.

BRASIL. Casa Civil. Lei no 13.146 de 6 de Julho de 2015. Brasília, DF, 2015. 40p.

CALADO, Jacqueline lukisa Faustino. et al. Emergência de Relações auditivo-visuais via treino por CRMTS para crianças com TEA. Acta Comportamentalia, Guadalajara, pp. 347362, set. 2018. Disponível em:

http://www.revistas.unam.mx/index.php/acom/article/view/66929/58827 Acesso em: 28 dez. 2018.

CALLOU, Isabela Cazuza; ASSIS, Grauben José Alves; BORBA, Marilu Michelly Cruz de. Controle Restrito de Estímulos e Autismo: Avaliação em tarefas de matching to sample com estímulos visuais. Acta Comportamentalia, Guadalajara, pp. 417-431, dez. 2018. Disponível em: http://www.revistas.unam.mx/index.php/acom/article/view/68120/60142 Acesso em: 28 dez. 2018.

CAPOVILLA, Fernando César. Filosofias Educacionais em Relação ao Surdo: Do Oralismo à Comunicação Total ao Bilinguismo. Revista Brasileira de Educação Especial, Bauru, pp. 99-116, 2000. Disponível em: 
http://dx.doi.org/10.5902/1984686X38150

https://www.abpee.net/homepageabpee04_06/artigos_em_pdf/revista6numero1pdf/r6_art06. pdf Acesso em: 2 mai. 2019.

CEDRO, Átila Moreira; PASSARELLI, Anna Christina P. M.; HUZIWARA, Edson Massayuki. Um panorama de estudos nacionais sobre a aquisição de nomeação em procedimentos com equivalência de estímulos e usuários de implante coclear. Revista Brasileira de Análise do Comportamento, Belém, pp. 84-96, jan 2014. Disponível em:

https://periodicos.ufpa.br/index.php/rebac/article/view/2552/2757 Acesso em: 28 dez 2018.

COZBY, Paul; BATES, Scott. Methods in Behaviorial Research. 11a ed. New York: McGraw-Hill, 2011.

DIAS, Katarina Kataoka; BARROS, Romariz da Silva. Classes Funcionais e de Equivalência em Crianças Diagnosticadas com Autismo. Revista Brasileira de Terapia

Comportamental e Cognitiva, São Paulo, pp.18-30, jan. 2017. Disponível em:

http://www.usp.br/rbtcc/index.php/RBTCC/article/view/947/495 Acesso em: 28 dez. 2018.

ELIAS, Nassim Chamel; ANGELOTTI, Vanessa Cristina. Ensino Informatizado de Frações para Crianças Surdas e Ouvintes. Acta Comportamentalia, Guadalajara, pp. 347-363, set. 2016. Disponível em:

http://www.revistas.unam.mx/index.php/acom/article/view/56968/50538 Acesso em: 28 dez. 2018

ESTEVES, Raquel Chaguri; LUCCHESI, Fernando Del Mando; ALMEIDA-VERDU, Ana Cláudia Moreira. Ensino de ecoico, tato e mando: uma revisão bibliográfica dos artigos o Journal of Applied Behavior Analysis (JABA). Revista Brasileira de Terapia

Comportamental e Cognitiva, São Paulo, pp. 109-124, jun. 2014. Disponível em:

http://www.usp.br/rbtcc/index.php/RBTCC/article/download/694/414/ Acesso em: 28 dz. 2018.

FERREIRA, Luciene Afonso; SILVA, Álvaro Júnior Melo; BARROS, Romariz da Silva.

Ensino da Aplicação de Tentativas Discretas a Cuidadores de Crianças Diagnosticada com Autismo. Revista Perspectivas em Análise do Comportamento, São Paulo, pp. 101-113, jan. 2016. Disponível em: http://pepsic.bvsalud.org/pdf/pac/v7n1/v7n1a08.pdf Acesso em: 28 dez. 2018.

FIALHO, Juliana Palma de Godoi; MICHELETTO, Nilza; SÉLIOS, Talita Lopes. Produção de Variabilidade Comportamental e sua Extensão em Crianças com Autismo. Acta

Comportamentalia, Guadalajara, pp. 391-404, dez. 2015. Disponível em:

http://www.revistas.unam.mx/index.php/acom/article/view/53792/47887 Acesso em: 28 dez. 2018.

GARCIA, Marcus Vinícius Fonseca de; OLIVEIRA, Thais Porlan. Redução de Comportamento Autolesivo em Criança com Diagnóstico de Autismo Utilizando Reforçamento Não Contingente e Treino de Mando. Revista Brasileira de Análise do Comportamento, Belém, pp.54-64, jan. 2016. Disponível em:

https://periodicos.ufpa.br/index.php/rebac/article/view/3790/0 Acesso em: 28 dez. 2018. 
http://dx.doi.org/10.5902/1984686X38150

GARCIA, Rafael Vilas Boas; ZAVITOSKI, Marina; ELIAS, Nassim Chamel. Avaliação intraverbal em pessoas com deficiência intelectual, autismo e desenvolvimento típico. Acta Comportamentalia, Guadalajara, pp. 233-247, mar. 2018. Disponível em: http://www.revistas.unam.mx/index.php/acom/article/view/65041/57071 Acesso em: 28 dez. 2018.

GAST, David L. Single Subject Research Methodology in Behavioral Sciences. 1. ed. New York: Routledge, 2010.

GOMES, Camila Graciela Santos; HANNA, Elenice Seixas; SOUZA, Deisy das Graças de. Ensino de Relações entre Figuras e Palavras Impressas com Emparelhamento Multimodelo a Crianças com Autismo. Revista Brasileira de Análise do Comportamento, Belém, pp. 2436, jan. 2015. Disponível em:

https://periodicos.ufpa.br/index.php/rebac/article/view/1975/3801 Acesso em: 28 dez. 2018.

GOULART, Paulo; ASSIS, Grauben José Alves. Estudos sobre autismo em análise do comportamento: aspectos metodológicos. Revista Brasileira de Terapia Comportamental e Cognitiva, São Paulo, pp 151-165, 2002. Disponível em:

http://pepsic.bvsalud.org/scielo.php?script=sci_arttext\&pid=S1517-55452002000200007 Acesso em: 28 dez 2018.

GUERRA, Bárbara Trevizan; ALMEIDA-VERDU, Ana Claudia Moreira. Ensino de operantes verbais em pessoas com transtorno do espectro autista no The Analysis of Verbal Behavior: Revisão Sistemática. Revista Brasileira de Terapia Comportamental e Cognitva, São Paulo, pp. 73-85, mar 2016. Disponível em:

http://www.usp.br/rbtcc/index.php/RBTCC/article/view/884/480 Acesso em: 28 dez 2018.

GUIMARÃES, Mariane Sarmento da Silva. et al. Treino de Cuidadores para Manejo de Comportamentos Inadequados de Crianças com Transtorno do Espectro do Autismo.

Revista Brasileira de Terapia Comportamental e Cognitiva, São Paulo, pp. 40-53, set. 2018. Disponível em: http://www.usp.br/rbtcc/index.php/RBTCC/article/view/1217/597 Acesso em: 28 dez. 2018.

HUSSEIN, Laila Guzzon. et al. Aquisição do comportamento de ouvir, baseada em seleção de figuras, em crianças com implante coclear contralateral. Revista Brasileira de Terapia Comportamental e Cognitiva, São Paulo, pp. 27-39, jan 2018. Disponível em: http://www.usp.br/rbtcc/index.php/RBTCC/article/view/1135/552 Acesso em: 28 dez 2018.

LUCCHESI, Fernando Del Mando. et al. Análise dos Passos de um Ensino Programado de Leitura e Escrita com Deficiência Auditiva e Implante Coclear. Acta Comportamentalia, Guadalajara, pp 137-151, mar 2015. Disponível em: http://www.revistas.unam.mx/index.php/acom/article/view/50103/45039 Acesso em: 28 dez 2018.

MACEDO, Marina; ESCOBAL, Giovana; GOYOS, Celso. Escolha e Preferência por Alimentos com ou sem Valor Calórico em Crianças com Deficiência Intelectual. Acta Comportamentalia, Guadalajara, pp. 83-98, jan. 2013. Disponível em: http://pepsic.bvsalud.org/pdf/actac/v21n1/a06.pdf Acesso em: 28 dez. 2018. 
MAGALHÃES, Priscila Gisela; ASSIS, Grauben Alves; ROSSIT, Rosana Aparecida. Emergência de Relações Monetárias por meio de Procedimento de Ensino de Escolha com o Modelo Por Resposta Construída para Crianças Surdas. Revista Brasileira de Terapia Comportamental e Cognitiva, São Paulo, pp. 35-55, mar. 2016. Disponível em: http://www.usp.br/rbtcc/index.php/RBTCC/article/view/882/478 Acesso em: 28 dez. 2018.

MALERBO, Alana C. D. Brunini; SCHMIDT, Andréia. Aprendizagem de Relações NomeTextura por meio de Dois Procedimentos de Ensino por Exclusão. Acta Comportamentalia, Guadalajara, pp. 5-23, jan. 2017. Disponível em: http://www.revistas.unam.mx/index.php/acom/article/view/58798/51939 Acesso em: 28 dez. 2018.

MARTES, Maria Carolina Côrrea; SANTOS-CARVALHO, Larissa Helena Zani. Uma revisão dos artigos publicados no Journal of Applied Behavior Analysis (JABA) sobre comportamento verbal e Autismo entre 2008 e 2012. Perspectiva em Análise do Comportamento, São Paulo, pp. 73-86, jun 2012. Disponível em: http://pepsic.bvsalud.org/pdf/pac/v3n2/v3n2a01.pdf Acesso em: 28 dez 2018.

MELO, Izabela Oliveira Bandeira de. et al. Efeitos de tarefas de exclusão e fading no ensino de relações auditivo-visuais. Acta Comportamentalia, Guadalajara, pp. 311-329, set 2018. Disponível em: http://www.revistas.unam.mx/index.php/acom/article/view/66916/58819 Acesso em: 28 dez 2018.

MESQUITA, Maria Luiza Guedes et al. Restrição Alimentar e Problemas de Comportamento de Crianças com Síndrome de Prader-Willi. Revista Brasileira de Terapia Comportamental e Cognitiva, São Paulo, pp. 30-40, jan. 2014. Disponível em: http://pepsic.bvsalud.org/pdf/rbtcc/v16n1/v16n1a04.pdf Acesso em: 28 dez. 2018.

MOHER, David; et al. Preferred Reporting Items for Systematic Reviews and Meta-Analyses: The PRISMA Statement. Annals of Internal Medicine, pp. 264-269, 2009. doi: 10.7326/0003-4819-151-4-200908180-00135

MURARI, Silvia Cristiane; MICHELETTO, Nilza. Avaliação de Comportamentos em Puericultura para Identificação Precoce do Transtorno do Espectro Autista. Revista Brasileira de Terapia Comportamental e Cognitiva, São Paulo, pp. 54-72, set. 2018. Disponível em: http://www.usp.br/rbtcc/index.php/RBTCC/article/download/1213/598/ Acesso em: 28 dez. 2018.

NICOLINO, Victor Faria; ZANOTTO, Maria Lourdes Bara. Revisão Histórica de Pesquisas em Análise do Comportamento e Educação especial/ Inclusão publicadas no JABA entre 2001 e 2008. Psicologia: Teoria e Pesquisa, Brasília, pp. 51-79, mar. 2010. Disponível em: http://pepsic.bvsalud.org/pdf/ptp/v12n2/v12n2a05.pdf Acesso em: 28 dez. 2018.

NICOLINO, Victor Faria; ZANOTTO, Maria de Lourdes Bara. Trabalhos de Análise do Comportamento na área da educação em periódicos brasileiros no período de 1961-2006. Acta Comportamentalia, Guadalajara, pp. 343-358, set 2011. Disponível em: http://pepsic.bvsalud.org/pdf/actac/v19n3/a06.pdf Acesso em: 28 dez 2018.

NICOLINO, Victor Faria; MALERBI, Fani Eta Korn. Promoção de Interações Sociais entre 
Colegas e Criança Autista em Ambiente de Inclusão. Acta Comportamentalia, Guadalajara, pp. 107-123, jan. 2011. Disponível em: http://pepsic.bvsalud.org/pdf/actac/v19n1/a06.pdf Acesso em: 28 dez. 2018.

NUNES, Leila Regina d'Oliveira de Paula. (Org.). Novas Trilhas no modo de fazer pesquisa em educação especial. 1. ed. São Carlos: Marquezine \& Manzini, 2014.

NUNES, Leila Regina d'Oliveira de Paula; WALTER, Cátia Crivelenti de Figueiredo. Pesquisa em Educação Especial. In: NUNES, Leila Regina d'Oliveira de Paula. (Org.). Novas Trilhas no modo de fazer pesquisa em educação especial. São Carlos: Marquezine \& Manzini, 2014. P. 27-51.

PASSARELLI, Anna Cristina Porto Maia. et al. Discriminação Auditivo-Visual com Pistas Orofaciais em Crianças Deficientes Auditivas. Acta Comportamentalia, Guadalajara, pp. 175-192, mar. 2013. Disponível em: http://pepsic.bvsalud.org/pdf/actac/v21n2/a03.pdf Acesso em: 28 dez. 2018.

PLÁCIDO, Thaís Texeira; MEDEIROS, Carlos Augusto; CRUZ, Gleidson Gabriel da. O Efeito de Instruções na Correspondência Verbal em Autista. Revista Brasileira de Terapia

Comportamental e Cognitiva, São Paulo, pp. 24-39, set. 2018. Disponível em: http://www.usp.br/rbtcc/index.php/RBTCC/article/view/1212/596 Acesso em: 28 dez. 2018.

PRISMA. Preferred Reporting Items for Systematic Reviews and Meta-Analyses.Transparent Reporting of Systematic Review and Meta-Analyses. 2009. 2p.

RESENDE, Alice Almeida Chaves; ELIAS, Nassim Chamel; GOYOS, Celso. Transferência de Funções Ordinais através de Classes de Estímulos Equivalentes em Surdos. Acta Comportamentalia, Guadalajara, pp. 317-326, set. 2012. Disponível em: http://pepsic.bvsalud.org/pdf/actac/v20n3/a05.pdf Acesso em: 28 dez. 2018.

ROCANTI, Ana Luiza; HÜBNER, Maria Martha Costa. Efeitos de Diferentes Magnitudes do Reforçador em Procedimento de Correção no Ensino de Respostas de Ouvinte e Falante em Crianças Diagnosticada com Autismo. Revista Brasileira de Terapia Comportamental e Cognitiva, São Paulo, pp. 9-23, set. 2018. Disponível em:

http://www.usp.br/rbtcc/index.php/RBTCC/article/view/1211/595 Acesso em: 28 dez. 2018.

RIQUE, Luciana Degrande. et al. Leitura após Formação de Classes de Equivalência em Crianças com Implante Coclear: Precisão e Fluência em Palavras e Textos. Acta

Comportamentalia, Guadalajara, pp. 301-327, set. 2017. Disponível em:

http://www.revistas.unam.mx/index.php/acom/article/view/61629/54288 Acesso em: 28 dez. 2018.

SANCHEZ, Cíntia Nazaré M.; GOUVEIA. Amauri. Adaptação da EAH para população de surdos falantes de Libras. Revista Brasileira de Terapia Comportamental e Cognitiva, São Paulo, pp. 171-179, mar 2008. Disponível em:

http://www.usp.br/rbtcc/index.php/RBTCC/article/view/186/154 Acesso em: 28 dez 2018.

SANCHEZ, Cíntia Nazaré M.; GOUVEIA, Amauri. O teste da simulação do falar em público não gera ansiedade em adolescente surdos ou ouvintes. Revista Brasileira de Terapia 
http://dx.doi.org/10.5902/1984686X38150

Comportamental e Cognitiva, São Paulo, pp. 21-32, mar 2011. Disponível em: http://pepsic.bvsalud.org/pdf/rbtcc/v13n2/v13n2a03.pdf Acesso em: 28 dez 2018.

SANTOS, Rafael Ernesto Arruda; ASSIS, Grauben José Alves; BORBA, Marilu Michelly Cruz da. Ensino de Discriminações Condicionais de Sentenças sobre a Emergência de Relações Sintáticas em Surdos. Perspectivas em Análise do Comportamento, São Paulo, pp. 86100, jan. 2016. Disponível em: http://pepsic.bvsalud.org/pdf/pac/v7n1/v7n1a07.pdf Acesso em: 28 dez. 2018.

SIDMAN, Murray. Equivalence relations and behavior: A research story. 1. ed. Boston: Authors Cooperative, 1994.

SIDMAN, Murray; TAILBY, William. Conditional Discrimination vs. matching to sample: an expansion of the testing paradigm. Journal of Experimental Analysis of Behavior, Columbia, pp. 5-22, jan. 1982. Disponível em:

https://www.ncbi.nlm.nih.gov/pmc/articles/PMC1333115/pdf/jeabehav00072-0007.pdf Acesso em: 30 dez 2018.

SILVA, Álvaro Júnior Melo e. et al. Efeitos de Números Exemplares sobre Aquisição e Generalidade de Desempenho em Crianças Diagnosticadas com Transtorno do Espectro Autista (TEA). Revista Brasileira de Análise do Comportamento, Belém, pp. 5-15, jan. 2017. Disponível em: https://periodicos.ufpa.br/index.php/rebac/article/view/5259/4436 Acesso em: 28 dez 2018.

SILVA, Raissa Viviane; NEVES, Anderson Jonas; ALMEIDA-VERDU, Ana Cláudia Moreira (2017). Ensino de Relações de Equivalência com Sentenças de Cinco Termos e Produção Oral em uma Criança com Implante Coclear. Acta Comportamentalia, Guadalajara, pp. 289306, set 2017. Disponível em:

http://www.revistas.unam.mx/index.php/acom/article/view/61628/54287 Acesso em: 28 dez 2018.

SOUZA, Fabiana Cristina de; ALMEIDA-VERDU, Ana Cláudia Moreira; BEVILACQUA, Maria Cecília. Ecoico e Nomeação de Figuras em Crianças com Deficiência Auditiva Pré-Lingual com Implante Coclear. Acta Comportamentalia, Guadalajara, pp. 325-339, set 2013. Disponível em: http://pepsic.bvsalud.org/pdf/actac/v21n3/a04.pdf Acesso em: 28 dez 2018.

SOUZA, Ruth Daisy Capistrano de. et al.. Emergência de Relações Ordinais sob Controle Contextual em Surdos. Revista Brasileira de Análise do Comportamento, Belém, pp. 3754, jan. 2010. Disponível em: https://periodicos.ufpa.br/index.php/rebac/article/view/993/1434 Acesso em: 28 dez 2018.

VARELLA, André A. B.; SOUZA, Deisy G. O Uso do Procedimento Blocado no Ensino de Discriminações Condicionais de Identidade para com Pessoas com Autismo: Efeitos do Emprego de Três Estímulos Modelos. Revista Brasileira de Análise do Comportamento, Belém, pp. 55-71, set 2011. Disponível em: https://periodicos.ufpa.br/index.php/rebac/article/view/1450/1833 Acesso em: 28 dez 2018. 
http://dx.doi.org/10.5902/1984686X38150

VELASCO, Saulo Missiaggia; GARCIA-MIJARES, Miriam; TOMANARI, Gerson Yukio. Fundamentos Metodológicos da Pesquisa em Análise Experimental do Comportamento. Psicologia em Pesquisa,Juiz de Fora, pp. 150-155, jul 2010. Disponível em: http://pepsic.bvsalud.org/pdf/psipesq/v4n2/v4n2a08.pdf Acesso em: 28 dez 2018.

\section{Notas}

${ }^{1}$ As filosofias educacionais sobre a surdez são o Oralismo, a Comunicação Total e o Bilinguismo (ver Capovilla, 2000).

\section{Correspondência}

Rafael Ernesto Arruda Santos - Universidade Federal de São Carlos, Rodovia Washington Luís, km 235 - SP - 310, São Carlos, São Paulo - Brasil.

CEP: 13565-905.

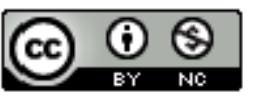

This work is licensed under a Creative Commons Attribution-NonCommercial 4.0 International (CC BY-NC 4.0)

Modalidade do artigo: Relato de pesquisa ( ) Revisão de Literatura (X) 